\title{
DMBA promotes ErbB2-mediated carcinogenesis via ErbB2 and estrogen receptor pathway activation and genomic instability
}

\author{
ZHIKUN MA ${ }^{1,2}$, YOUNG MI KIM ${ }^{3}$, ERIN W. HOWARD ${ }^{4}$, XIAOSHAN FENG ${ }^{2}$, STANLEY D. KOSANKE $^{1}$, \\ SHIHE YANG ${ }^{1}$, YUNBO JIANG $^{1}$, AMANDA B. PARRIS $^{4}, \mathrm{XIACAO}^{4}, \mathrm{SHIBO} \mathrm{LI}^{3}$ and XIAOHE YANG ${ }^{1,2,4}$ \\ ${ }^{1}$ Department of Pathology, University of Oklahoma Health Sciences Center, Oklahoma City, OK 73104, USA; \\ ${ }^{2}$ Department of Oncology, First Affiliated Hospital of Henan University of Sciences and Technology, Luoyang, \\ Henan 471500, P.R. China; ${ }^{3}$ Department of Pediatrics, University of Oklahoma Health Sciences Center, \\ Oklahoma, OK 73104; ${ }^{4}$ Julius L. Chambers Biomedical/Biotechnology Research Institute, \\ Department of Biological and Biomedical Sciences, North Carolina Central University, \\ North Carolina Research Campus, Kannapolis, NC 28081, USA
}

Received January 22, 2018; Accepted June 21, 2018

DOI: $10.3892 /$ or.2018.6545

\begin{abstract}
Environmental factors, including 7,12-dimethylbenz [a]anthracene (DMBA) exposure, and genetic predisposition, including ErbB2 overexpression/amplification, have been demonstrated to increase breast cancer susceptibility. Although DMBA- and ErbB2-mediated breast cancers are well-studied in their respective models, key interactions between environmental and genetic factors on breast cancer risk remain unclear. Therefore, the present study aimed to investigate the effect of DMBA exposure on ErbB2-mediated mammary tumorigenesis. MMTV-ErbB2 transgenic mice exposed to DMBA (1 mg) via weekly oral gavage for 6 weeks exhibited significantly enhanced mammary tumor development, as indicated by reduced tumor latency and increased tumor multiplicity compared with control mice. Whole mount analysis of premalignant mammary tissues from 15-week-old mice revealed increased ductal elongation and proliferative index in DMBA-exposed mice. Molecular analyses of premalignant mammary tissues further indicated that DMBA exposure enhanced epidermal growth factor receptor (EGFR)/ErbB2 and estrogen receptor (ER) signaling, which was associated with increased mRNA levels of EGFR/ErbB2 family members and ER-targeted genes. Furthermore, analysis of tumor karyotypes revealed that DMBA-exposed tumors displayed
\end{abstract}

Correspondence to: Dr Xiaohe Yang, Julius L. Chambers Biomedical/Biotechnology Research Institute, Department of Biological and Biomedical Sciences, North Carolina Central University, North Carolina Research Campus, 500 Laureate Way, Kannapolis, NC 28081, USA

E-mail: xyang@nccu.edu

Key words: 7,12-dimethylbenz[a]anthracene, MMTV-ErbB2 transgenic mice, breast cancer, genomic instability, receptor tyrosine kinase pathway, estrogen receptor pathway more chromosomal alterations compared with control tumors, implicating DMBA-induced chromosomal instability in tumor promotion in this model. Together, the data suggested that DMBA-induced deregulation of EGFR/ErbB2-ER pathways plays a critical role in the enhanced chromosomal instability and promotion of ErbB2-mediated mammary tumorigenesis. The study highlighted gene-environment interactions that may increase risk of breast cancer, which is a critical clinical issue.

\section{Introduction}

Increasing evidence indicates that interactions between environmental factors and genetic predisposition play a critical role in cancer development and progression (1-4). In particular, polycyclic aromatic hydrocarbon $(\mathrm{PAH})$ compounds are a group of common environmental pollutants with carcinogenic effects (5-7). 7,12-dimethylbenz[a]anthracene (DMBA), a well-studied carcinogen, is a prototypical member of the PAH family that has been extensively used to induce the development of mammary and other tumor types in preclinical animal models (8-10). Although PAHs have been studied in multiple cancer models, the general understanding of the specific genetic factors that contribute to PAH-induced cancer susceptibility remains limited. Therefore, clinically relevant mechanistic studies will provide insight into cancer-promoting gene-environment interactions, which will be of pivotal significance in breast cancer etiology and prevention.

Previous studies suggest that DMBA-mediated carcinogenesis involves DNA damage and the deregulation of genes critical for cell proliferation and survival (11). It has also been reported that DMBA activates the aryl hydrocarbon receptor (AhR), a transcription factor that regulates a number of genes involved in cellular metabolism (9). AhR-dependent upregulation of cytochrome P450 enzymes metabolizes DMBA into a mutagenic intermediate that causes DNA damage and subsequently contributes to the initiation of tumorigenesis (12,13). DMBA-mediated upregulation of Cyclin D1 and $\mathrm{c}-\mathrm{Myc}$, possibly through nuclear factor- $\kappa \mathrm{B}$ and Wnt pathways, 
has also been revealed to play a critical role in carcinogenesis $(11,14)$. Results from microarray gene expression profiling of mammary tissues from DMBA-treated rats indicated that DMBA alters genes involved in major cellular processes, including cellular differentiation, proliferation/cell cycle regulation, and microtubule dynamics (14), suggesting that DMBA-mediated carcinogenesis has broad-reaching effects on signaling transduction and gene expression. Therefore, it is of vital importance to study the impact of DMBA exposure on the specific mechanisms that modulate these pathways. In particular, the association of DMBA with clinically relevant genetic predisposition may be a critical factor that influences DMBA- and other PAH-associated breast cancer risk.

In regards to established models of genetic predisposition for breast cancer, ErbB2 is an oncogene overexpressed/amplified in approximately one-third of invasive breast cancers $(15,16)$. ErbB2 overexpression is also associated with poor prognosis and therapeutic resistance (17). As a member of the epidermal growth factor receptor (EGFR) family of receptor tyrosine kinases (RTKs), ErbB2 activates downstream pathways, such as the phosphoinositide-3-kinase (PI3K)/RAC-alpha serine/threonine-protein kinase (Akt) and mitogen-activated protein kinase (MAPK)/extracellular signal-regulated kinase (Erk) pathways that promote cell proliferation and survival. Deregulation of these signaling pathways can induce malignant transformation and tumor progression (18). Although ErbB2 overexpression alone is oncogenic, ErbB2-mediated carcinogenesis can be modulated by various environmental factors. Therefore, animal models of ErbB2-overexpressing human breast cancers have been developed as clinically relevant models for studying gene-environment interactions that influence breast cancer risk. Of particular importance to the present study, MMTV-ErbB2 transgenic mice are a well-established model used for testing the impact of various factors on breast cancer risk (19-22). Previous studies demonstrate that mammary tumor development in MMTV-ErbB2 mice is significantly modulated by various etiological factors (23-26). As such, MMTV-ErbB2 mice are ideal for studying breast cancer etiology due to long tumor latency with the development of spontaneous, nodular mammary tumors around 36 weeks of age. Given the well-defined genetic background and pathology of mammary tumor development in MMTV-ErbB2 mice $(14,27,28)$, this animal model has significant advantages for mechanistic studies involving gene-environment interactions.

In the present study, the effects of DMBA on mammary tumor development, as well as the underlying mechanisms, were investigated in MMTV-ErbB2 mice. It was demonstrated that DMBA promoted ErbB2-mediated carcinogenesis through the enhanced activation of ErbB2 and estrogen receptor (ER) signaling and the induction of further genomic instability. Overall, the study further supports the use of the MMTV-ErbB2 transgenic mouse model for investigating gene-environment interactions that are associated with breast carcinogenesis.

\section{Materials and methods}

Animals and treatment. Female FVB/N-Tg/MMTV-ErbB2 (MMTV-ErbB2) transgenic mice (weighing 15-20 g) were purchased from Jackson Laboratory (Bar Harbor, ME, USA).
Mice were fed an estrogen-free AIN-93G semi-purified diet (Bio-Serv; Flemington, NJ, USA) and provided water ad libitum. Mice were housed in an environment with an ambient temperature of $22 \pm 2^{\circ} \mathrm{C}$, relative humidity of $50 \pm 10 \%$, and a $12 \mathrm{~h}$ light/dark cycle. A total of 52 female mice were randomly assigned to either control or DMBA groups $(n=26$ mice per group). Beginning at 6 weeks of age, mice were treated weekly by oral gavage with $1 \mathrm{mg}$ DMBA in $0.1 \mathrm{ml}$ peanut oil or an equal volume of peanut oil alone (vehicle) for 6 weeks. The DMBA dose was based on previous studies using the parental FVB/N mouse strain (11). All animal procedures were approved by the University of Oklahoma Health Sciences Center Institutional Animal Care and Use Committee (Oklahoma City, OK, USA).

Starting at 10 weeks of age, mice were examined for mammary tumors twice a week. The dates of the first palpable mammary tumors were recorded for each mouse. When palpable mammary tumors reached a volume of $1.5 \mathrm{~cm}^{3}$, mice were euthanized by carbon dioxide asphyxiation followed by cervical dislocation. Then, the tumor and lung tissues were harvested for molecular and histological analyses. For histopathological analysis, the collected tissues were fixed overnight $(\sim 18 \mathrm{~h})$ in $10 \%$ neutral formalin at room temperature and paraffin-embedded (FFPE). Tumor and lung tissue sections (5 $\mu \mathrm{m}$ thickness) were deparaffinized in xylene and hydrated in 5 min washes of 100, 95 and $70 \%$ ethanol. Then, sections were hematoxylin and eosin (H\&E)-stained with hematoxylin for $5 \mathrm{~min}$ and eosin $\mathrm{Y}$ for $1 \mathrm{~min}$ at room temperature. Finally, the sections were dehydrated with $5 \mathrm{~min}$ washes of $70 \%, 95 \%$, and $100 \%$ ethanol, cleared with xylene, and mounted with Permount. Tumor latency and tumor multiplicity were calculated at the endpoint of the experiment (when all mice had developed tumors).

Mammary whole mounts. At 15 weeks of age, 6 mice from each group were euthanized and the abdominal mammary glands were removed and spread onto glass slides for overnight fixation ( $\sim 18 \mathrm{~h})$ in Carnoy's solution (6:3:1 ratio of $100 \%$ ethanol:chloroform:glacial acetic acid) at room temperature. Then, the slides were washed in $70 \%$ ethanol, rehydrated in a series of $50 \%$ and $30 \%$ ethanol solutions (in distilled water), and followed by staining for $12 \mathrm{~h}$ in carmine alum at room temperature. Mammary glands were then dehydrated with $70 \%, 95 \%$, and $100 \%$ ethanol and cleared in xylene before mounting with Permount. Whole mounts were imaged using a Nikon Eclipse inverted microscope (Nikon DXM1200c; Nikon Corporation, Tokyo, Japan) with Nikon Elements Imaging System (Nikon Corporation). The ductal extension beyond the lymph node was measured.

5-Bromo-2'-deoxyuridine (BrdU) incorporation and immunohistochemistry (IHC). For the BrdU incorporation assay, 15-week-old control and DMBA-exposed mice were intraperitoneally injected with $\mathrm{BrdU}(3 \mathrm{mg} / \mathrm{ml}$ in $0.1 \mathrm{ml}) 2 \mathrm{~h}$ prior to euthanization. Then, the inguinal mammary gland tissues were collected. The FFPE tissue sections were deparaffinized with xylene and hydrated in 5 min washes of $100 \%, 95 \%$, and $70 \%$ ethanol. For antigen retrieval, the tissue sections were boiled in citrate buffer ( $\mathrm{pH} 6.0$ ) at $100^{\circ} \mathrm{C}$ for $30 \mathrm{~min}$, then DNA was denatured with $\mathrm{HCl}$ at $37^{\circ} \mathrm{C}$ for $30 \mathrm{~min}$. Endogenous 
Table I. Primary antibodies used for western blotting.

\begin{tabular}{|c|c|c|c|}
\hline Antibody name & Dilution & Vendor & Catalog \# \\
\hline EGF Receptor (D38B1) XP Rabbit mAb & $1: 1,000$ & Cell Signaling Technology & 4267 \\
\hline Phospho-EGF Receptor (Tyr1068) (D7A5) XP Rabbit mAb & $1: 1,000$ & Cell Signaling Technology & 3777 \\
\hline HER2/ErbB2 (29D8) Rabbit mAb & $1: 1,000$ & Cell Signaling Technology & 2165 \\
\hline Phospho-HER2/ErbB2 (Tyr877) Antibody & $1: 1,000$ & Cell Signaling Technology & 2241 \\
\hline HER3/ErbB3 (D22C5) XP Rabbit mAb & $1: 1,000$ & Cell Signaling Technology & 12708 \\
\hline Akt1 (C73H10) Rabbit mAb & $1: 2,000$ & Cell Signaling Technology & 2938 \\
\hline Phospho-Akt (Ser473) (D9E) XP Rabbit mAb & $1: 2,000$ & Cell Signaling Technology & 4060 \\
\hline ERK2 Antibody (C-14) & $1: 2,000$ & Santa Cruz Biotechnology & sc-154 \\
\hline Phospho-p44/42 MAPK (Erk1/2) (Thr202/Tyr204) Antibody & $1: 2,000$ & Cell Signaling Technology & 9101 \\
\hline$\beta$-Actin Antibody (C4) & $1: 3,000$ & Santa Cruz Biotechnology & sc-47778 \\
\hline ER $\alpha$ Antibody (MC-20) & $1: 2,000$ & Santa Cruz Biotechnology & sc-542 \\
\hline Phospho-Estrogen Receptor $\alpha$ (Ser167) (D5W3Z) Rabbit mAb & $1: 1,000$ & Cell Signaling Technology & 64508 \\
\hline ER $\beta$ Antibody (H-150) & $1: 1,000$ & Santa Cruz Biotechnology & sc-8974 \\
\hline c-Myc Antibody (9E10) & $1: 2,000$ & Santa Cruz Biotechnology & sc- 40 \\
\hline Cyclin D1 Antibody (HD11) & $1: 2,000$ & Santa Cruz Biotechnology & sc-246 \\
\hline Bcl-2 Antibody (C-2) & $1: 2,000$ & Santa Cruz Biotechnology & sc-7382 \\
\hline
\end{tabular}

peroxidases were blocked by $3 \%$ hydrogen peroxide at room temperature for $10 \mathrm{~min}$. Non-specific binding was blocked by $10 \%$ horse serum (Vector Laboratories; Burlingame, CA, USA) for $1 \mathrm{~h}$ at room temperature, followed by incubation with the primary antibody against $\mathrm{BrdU}$ (1:1,000 dilution; Bu20a Mouse mAb; Cat. \#5292; Cell Signaling Technology, Danvers, MA, USA) at $4^{\circ} \mathrm{C}$ overnight and biotinylated anti-mouse secondary antibody (1:200 dilution; Vectastain ABC kit; Cat. \#PK-6102; Vector Laboratories) for $30 \mathrm{~min}$ at room temperature. The sections were exposed to $\mathrm{ABC}$ reagent (Vectastain $\mathrm{ABC}$ kit, Vector Laboratories) for $30 \mathrm{~min}$ at room temperature prior to staining with diaminobenzidine (DAB) for $1 \mathrm{~min}$ at room temperature and counterstaining with hematoxylin for about $1.5 \mathrm{~min}$ at room temperature. Subsequently, the sections were dehydrated with 5 min washes at room temperature of $70 \%$, 95\%, and $100 \%$ ethanol, cleared with xylene, and mounted with Permount for observation. Side branch ductal structures were analyzed in each sample using a Nikon Eclipse inverted microscope (x400 total magnification; Nikon Corporation) to determine the percentage of epithelial cells that exhibited $\mathrm{BrdU}^{+}$staining.

Western blotting. Mammary gland tissues were collected at 15 weeks of age and snap-frozen in liquid nitrogen prior to lysate preparation. The tissues were homogenized at $4^{\circ} \mathrm{C}$ in NP40-based cell lysis buffer $(50 \mathrm{mM}$ Tris $\bullet \mathrm{HCl}, \mathrm{pH} 7.6$, $150 \mathrm{mM} \mathrm{NaCl}, 1 \% \mathrm{NP}-40,1 \%$ sodium deoxycholate, $0.1 \%$ SDS) supplemented with protease and phosphatase inhibitor cocktail (Thermo Fisher Scientific, Inc., Waltham, MA, USA) and centrifuged at $18,000 \mathrm{xg}$ for $20 \mathrm{~min}$ at $4^{\circ} \mathrm{C}$. The proteins in the supernatant were collected and quantified using a BCA Protein Assay kit (Thermo Fisher Scientific, Inc.). Total protein $(50 \mu \mathrm{g})$ from each sample was separated by 10-12\% SDS-PAGE and transferred onto nitrocellulose membranes. Following blocking in 5\% non-fat milk in Tris-buffered saline/Tween-20 (TBST) for $1 \mathrm{~h}$ at room temperature, membranes were incubated with the indicated primary antibodies (Table I) overnight at $4^{\circ} \mathrm{C}$, followed by washing with TBST. After incubation with horseradish peroxidase-linked secondary antibodies (1:5,000 dilution; anti-mouse IgG or anti-rabbit IgG; Cat. \#7076 or \#7074; Cell Signaling Technology) for $1 \mathrm{~h}$ at room temperature and washing with TBST, specific protein bands were visualized with SuperSignal West Pico Chemiluminescent solution (Thermo Fisher Scientific, Inc.). Protein bands were imaged using the FluorChemE imaging system.

Reverse transcription-quantitative polymerase chain reaction ( $R T-q P C R)$. Total RNA was extracted from the inguinal mammary glands using an RNeasy Mini kit (Qiagen, Inc.; Valencia, CA, USA) according to the manufacturer's protocol. cDNA was synthesized using $1 \mu \mathrm{g}$ of RNA and TaqMan Reverse Transcription Reagents (Applied Biosystems; Thermo Fisher Scientific, Inc.) at $25^{\circ} \mathrm{C}$ for $10 \mathrm{~min}, 37^{\circ} \mathrm{C}$ for $30 \mathrm{~min}$ and $95^{\circ} \mathrm{C}$ for $5 \mathrm{~min}$. qPCR was performed using a Bio-Rad CFX1000 Real-Time PCR System (Bio-Rad Laboratories, Inc., Hercules, CA, USA). PCR amplification was carried out in a $20 \mu 1$ reaction volume containing $50 \mathrm{ng}$ cDNA, $0.4 \mu \mathrm{M}$ each forward and reverse primers (Table II), and $10 \mu \mathrm{l}$ SsoFast EvaGreen Supermix (Bio-Rad Laboratories). PCR reactions were initiated with denaturation at $95^{\circ} \mathrm{C}$ for $30 \mathrm{sec}$, followed by 39 amplification cycles at $95^{\circ} \mathrm{C}$ for $5 \mathrm{sec}$ and $60^{\circ} \mathrm{C}$ for $5 \mathrm{sec}$. Relative mRNA levels were quantified using the $2^{-\Delta \Delta \mathrm{Ca}}$ method (29) based on the cycle threshold values and were normalized to $\beta$-actin gene expression.

Chromosome preparation and karyotyping. Chromosomal isolation and karyotyping were performed according to the authors' previous study (30). Briefly, tumors were removed from MMTV-ErbB2 mice ( $\mathrm{n}=6$ mice per treatment) when tumor volumes reached $1.5 \mathrm{~cm}^{3}$. Then, tumor tissues were processed and cultured in DMEM/F12 media (Gibco; Thermo Fisher Scientific, Inc.) supplemented with $10 \%$ fetal bovine serum 
Table II. Primers used for reverse transcription-quantitative polymerase chain reaction.

\begin{tabular}{ll}
\hline Gene name & \multicolumn{1}{c}{ Sequence (5'-3') } \\
\hline$E G F R$ & \\
$\mathrm{~F}$ & TGGTAAGTCAGGGGCAAGTC \\
$\mathrm{R}$ & ACATGGCACTTCCTGGTGAT \\
$E R B B 2$ & \\
$\mathrm{~F}$ & CAGCCCCAGAGGATTACAGA \\
$\mathrm{R}$ & TCAGTCCTAGTGGGGTGTCC \\
$E R B B 3$ & \\
$\mathrm{~F}$ & GAGCTTCCAGACTCCGTTTG \\
$\mathrm{R}$ & AAATGGCCTGCAGCTTACAC \\
$E S R 1$ & \\
$\mathrm{~F}$ & TCTCTGGAAGAGAAGGACCACATC \\
$\mathrm{R}$ & TGCAGAGTCAGGCCAGCTTT \\
$M Y C$ & \\
$\mathrm{~F}$ & TGAGCCCCTAGTGCTGCAT \\
$\mathrm{R}$ & AGCCCGACTCCGACCTCTT \\
$C C N D 1$ & TGGAATCCTGTGGCATCCATGAAA C \\
$\mathrm{F}$ & GGGCACCTGGATTGTTCT \\
$\mathrm{R}$ & CACCGGAGACTCAGAGCA \\
$J U N$ & AAAACCTTGAAAGCGCAAAA \\
$\mathrm{F}$ & GTT TGCAACTGCTGCGTTAG \\
$\mathrm{R}$ & \\
$\mathrm{F}$ & \\
$\mathrm{R}$ & \\
\hline
\end{tabular}

$\mathrm{F}$, forward; R, reverse.

(Atlanta Biologicals; Flowery Branch, GA, USA) and penicillin/streptomycin. After the second passage, the tumor cells were collected following cell cycle arrest at metaphase, which was induced using Colcemid (final concentration: $0.02 \mu \mathrm{g} / \mathrm{ml}$; Gibco; Thermo Fisher Scientific, Inc.) for $1 \mathrm{~h}$. Trypsin-Giemsa banding (GTG banding) was used to treat and stain the chromosomes in the tumor cells. Tumor cells were exposed to Giemsa stain for $5 \mathrm{~min}$ at room temperature. At least 18 cells in metaphase were karyotyped for each tumor sample.

Statistical analysis. The Kaplan-Meier tumor-free survival curve was analyzed using a log-rank test to compare tumor latency between the control and DMBA-treated groups (GraphPad Prism; GraphPad Software, Inc., La Jolla, CA, USA) $(24,31)$. For all other analyses, significant differences between the means of two groups were examined using two-sample Student's t-tests (GraphPad Prism) $(32,33)$. All samples were analyzed in at least triplicate. $\mathrm{P}<0.05$ was considered to indicate a statistically significant difference.

\section{Results}

DMBA promotes mammary tumorigenesis in MMTV-ErbB2 transgenicmice.Totest the effects of DMBA onErbB2-mediated mammary tumorigenesis, MMTV-ErbB2 transgenic mice were exposed to DMBA for 6 weeks. In the control group, palpable mammary tumors first appeared at 24 weeks of age with an average latency of $37.7 \pm 2.3$ weeks (Fig. 1A). In contrast, all mice in the DMBA-exposed group developed palpable mammary tumors between 16 and 26 weeks of age with an average latency of $19.7 \pm 0.6$ weeks. By the experimental endpoint (when tumors reached $1.5 \mathrm{~cm}^{3}$ ), the average number of palpable tumors per mouse in the control group was $1.15 \pm 0.08$ tumors, while the average tumor multiplicity in the DMBA-exposed mice was $2.35 \pm 0.3$ tumors (Fig. 1B). As such, the total number of tumors was 23 in control-treated mice and 47 tumors in DMBA-exposed mice. Since the lungs are a relatively common metastatic site in MMTV-ErbB2 mice, the present study examined the frequency of mice with lung metastasis when mammary tumors reached $1.5 \mathrm{~cm}^{3}$. Metastases in lung tissues (Fig. 1C) were detected in 6 of 15 DMBA-treated mice $(40 \%)$ compared with only one mouse with lung metastasis $(5 \%)$ in the control group. IHC analysis confirmed that the lung metastases were ErbB2-overexpressing and derived from the primary mammary tumors rather than primary lung tumors (data not shown). Taken together, these results demonstrated that DMBA exposure significantly accelerated mammary tumor development, increased tumor multiplicity, and promoted tumor metastasis to the lungs in MMTV-ErbB2 transgenic mice.

DMBA alters mammary morphogenesis in MMTV-ErbB2 mice. To study the effects of DMBA on mammary tissues prior to malignant transformation, the present study examined the morphology and histopathology of the premalignant mammary glands of 15-week-old control and DMBA-treated mice. It was observed that DMBA markedly modified mammary morphogenesis as characterized by distinct ductal growth and lateral branching patterns (Fig. 2A). Mammary glands from 15-week-old control mice displayed shorter ductal elongation compared with DMBA-exposed mice (5.5 \pm 0.4 vs. $15.5 \pm 0.8 \mathrm{~mm}$, respectively), but relatively more complex ductal branching (Fig. 2B). Notably, DMBA exposure increased the presence of terminal end bud (TEB)-like structures, which were not evident in the 15 -week-old control mice. IHC analysis of BrdU nuclear incorporation further demonstrated that DMBA significantly increased the percentage of proliferating cells as compared to the mammary tissues from control MMTV-ErbB2 mice (Fig. 2C). Despite the observed differences in mammary morphogenesis, histopathological analysis of H\&E-stained mammary tumors indicated single nodular tumors in both control and DMBA-exposed mice (Fig. 2D), which was consistent with previous reports in MMTV-ErbB2 mice $(11,34)$. Together, these data suggest that the promotion of ErbB2-mediated mammary tumorigenesis by DMBA is associated with mammary gland developmental changes in MMTV-ErbB2 mice.

$D M B A$ enhances the activation of RTK signaling in mammary tissues from MMTV-ErbB2 mice. To understand the tumor-promoting molecular mechanisms of DMBA in MMTV-ErbB2 mice, the present study examined the effects of DMBA on RTK signaling and downstream targets in the premalignant mammary glands. As presented in Fig. 3A, 

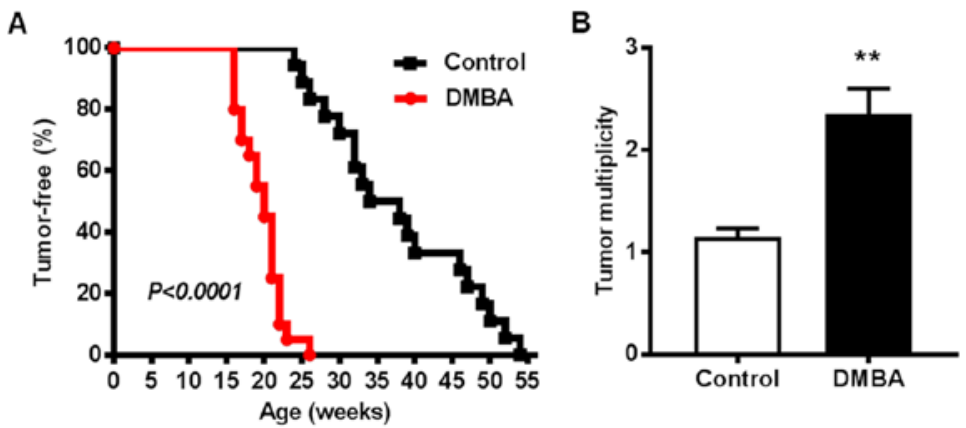

C

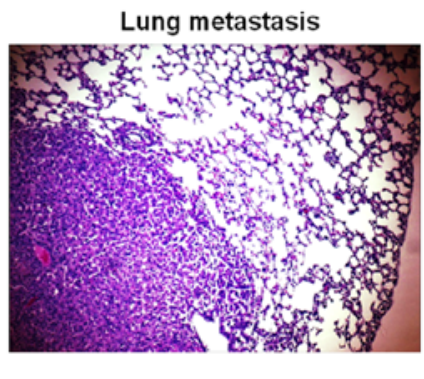

Figure 1. DMBA promotes mammary tumorigenesis in MMTV-ErbB2 transgenic mice. MMTV-ErbB2 mice were treated weekly by oral gavage with vehicle $(0.1 \mathrm{ml}$ peanut oil) or $1 \mathrm{mg}$ DMBA in $0.1 \mathrm{ml}$ peanut oil for 6 weeks until 12 weeks of age. (A) Tumor latency and (B) multiplicity were recorded in control and DMBA-exposed MMTV-ErbB2 mice ( $\mathrm{n}=20$ mice/group). The average tumor multiplicity is graphed \pm standard error. ${ }^{* *} \mathrm{P}<0.01 \mathrm{vs}$. control. (C) A representative image of hematoxylin and eosin-stained metastatic lung tissue from a DMBA-treated MMTV-ErbB2 mouse (magnification, x100). DMBA, 7,12-dimethylbenz[a]anthracene.

A
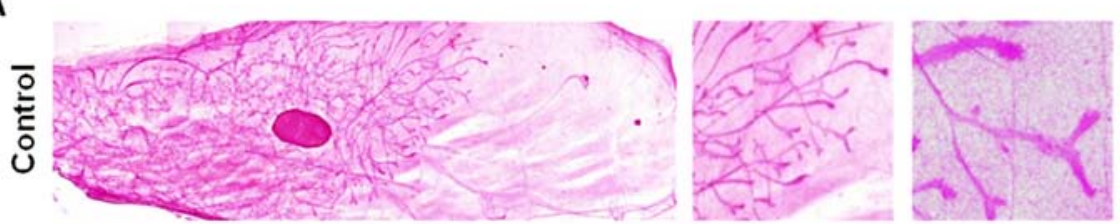
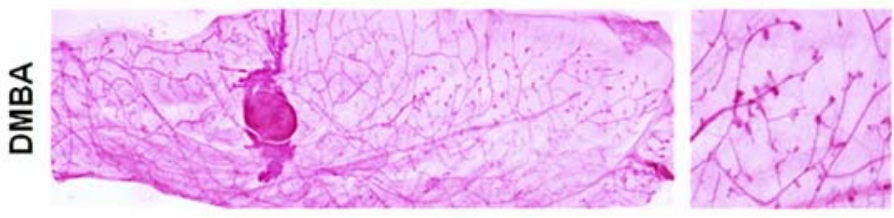

B

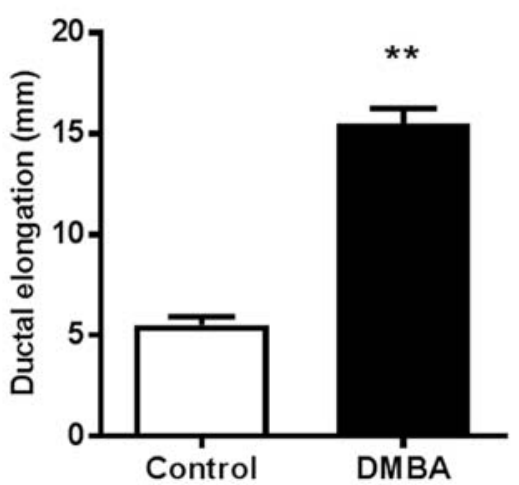

C
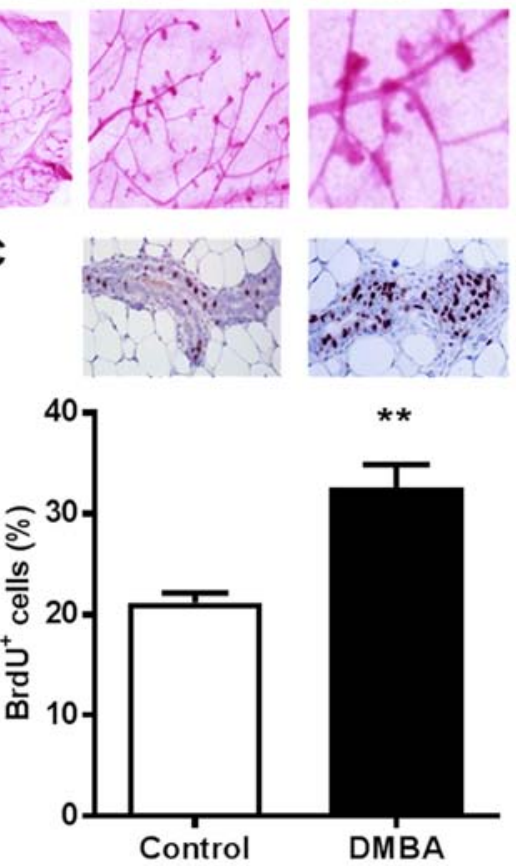

D

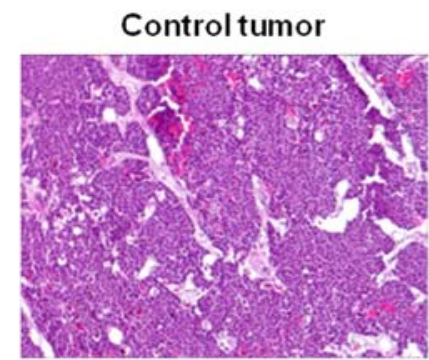

DMBA tumor

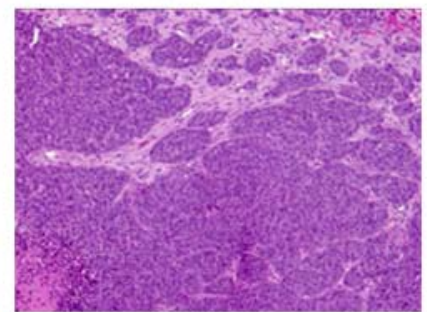

Figure 2. DMBA alters mammary morphogenesis in MMTV-ErbB2 mice. Mammary tissues were collected from 15-week-old control and DMBA-treated MMTV-ErbB2 mice ( $\mathrm{n}=6$ mice/group for whole mount and BrdU incorporation analyses). (A) Representative images of mammary whole mounts are shown at increasing magnifications (1x, 2x, and 6x magnifications). (B) Average ductal extension beyond the lymph node measured in the mammary whole mounts. (C) Average percentage of BrdU cells is graphed based on BrdU incorporation examined by immunohistochemistry in control and DMBA-exposed mammary glands. Representative images are shown above the graph with brown-staining indicating BrdU incorporation in the proliferating cells (magnification, $\mathrm{x} 400$ ). (D) Hematoxylin and eosin-stained tumor sections from control (Control tumor) and DMBA-treated (DMBA tumor) MMTV-ErbB2 mice are displayed (magnification, $\mathrm{x} 100)$. Data are presented as the mean \pm standard error. ${ }^{* *} \mathrm{P}<0.01$ vs. control. DMBA, 7,12-dimethylbenz[a]anthracene; BrdU, 5-bromo-2'-deoxyuridine. 
A

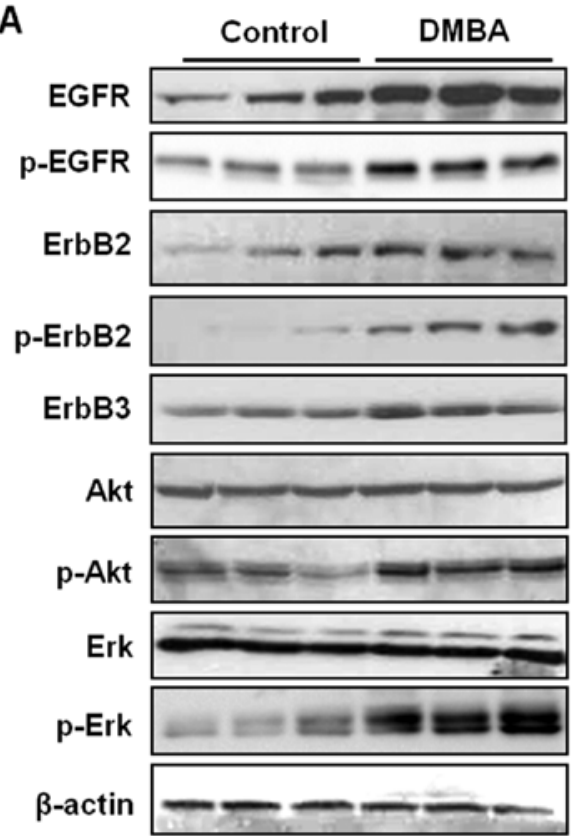

B

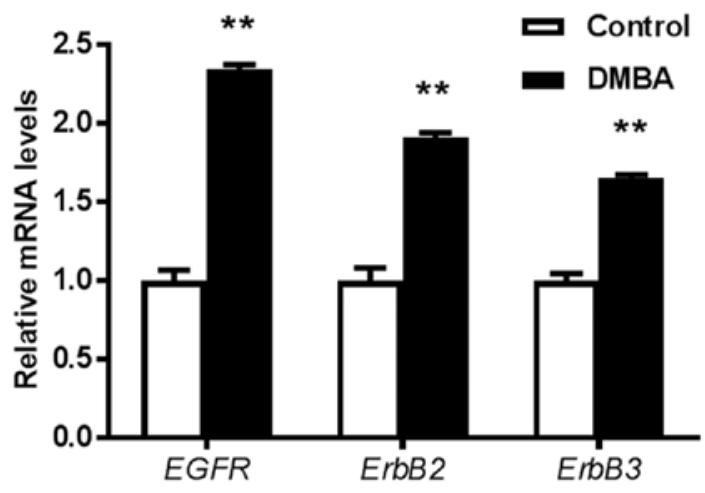

Figure 3. DMBA enhances the activation of RTK signaling in premalignant mammary tissues from MMTV-ErbB2 mice. Protein and RNA lysates were collected from the mammary glands of 15 -week-old control and DMBA-treated mice ( $\mathrm{n}=3$ mice/group). (A) Expression levels of total and phosphorylated protein markers of RTK pathways were detected using western blotting. (B) The relative mRNA levels for the indicated genes were measured using reverse transcription-quantitative polymerase chain reaction. Data are presented as the mean \pm standard error. ${ }^{* *} \mathrm{P}<0.01$ vs. control. DMBA, 7,12-dimethylbenz[a] anthracene; BrdU, 5-bromo-2'-deoxyuridine; Akt, RAC-alpha serine/threonine-protein kinase; EGFR, epidermal growth factor receptor; Erk, extracellular signal-regulated kinase; $\mathrm{p}$, phosphorylated.

A

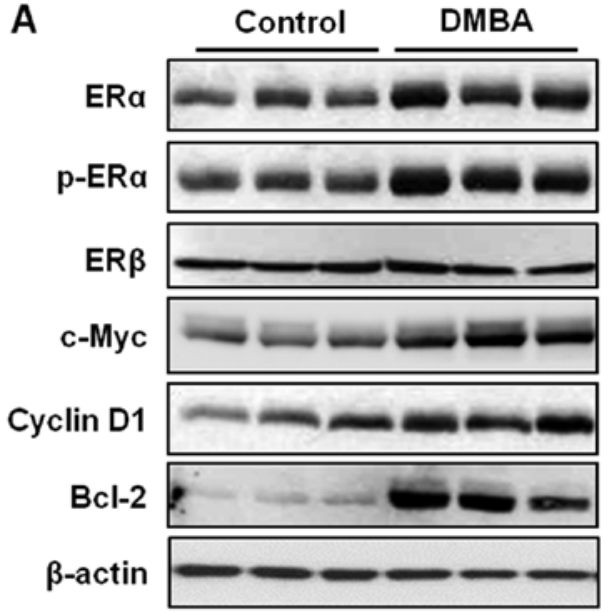

B

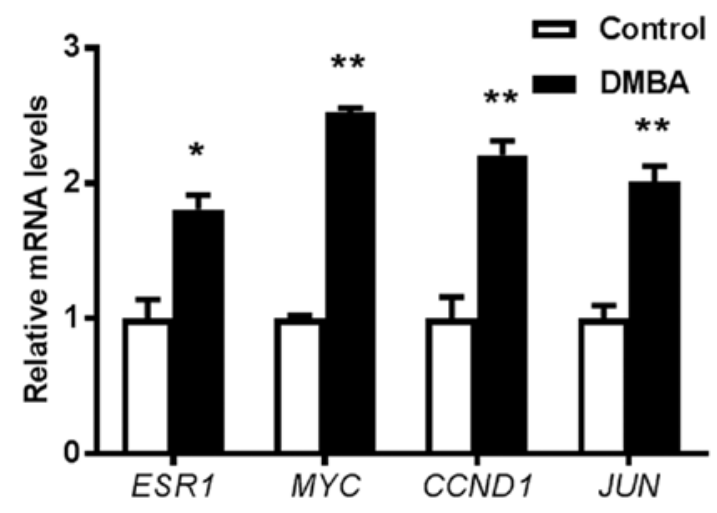

Figure 4. DMBA promotes ER signaling in premalignant mammary tissues from MMTV-ErbB2 mice. Protein and RNA lysates were collected from the mammary glands of 15 -week-old control and DMBA-treated mice ( $\mathrm{n}=3$ mice/group). (A) Expression levels of total and phosphorylated protein markers of the ER pathway were detected using western blotting. (B) The relative mRNA levels for the indicated genes were measured using reverse transcription-quantitative polymerase chain reaction. Data are presented as the mean \pm standard error. ${ }^{*} \mathrm{P}<0.05,{ }^{* *} \mathrm{P}<0.01$ vs. control. DMBA, 7,12-dimethylbenz[a]anthracene; ER, estrogen receptor; Bcl-2, B cell lymphoma-2; ESR1, ER $\alpha$; MYC, c-Myc; CCND1, Cyclin D1; JUN, c-Jun.

DMBA significantly increased the activation/phosphorylation of EGFR, ErbB2, Akt, and Erk in the premalignant mammary tissues from 15-week-old DMBA-treated mice, compared with control mice. This indicated that DMBA amplified the activation of ErbB2/MAPK/PI3K/Akt signaling, which is the driving force of ErbB2-mediated carcinogenesis. It was also revealed that the mRNA levels of $E G F R, E R B B 2$, and $E R B B 3$ were increased by DMBA (Fig. 3B). To note, the mRNA expression of the ERBB2 transgene was not significantly affected by DMBA exposure (data not shown). Together, these data suggest that the augmented upregulation of RTKs is an important mechanism that contributes to DMBA-promoted mammary tumor development in MMTV-ErbB2 mice.

$D M B A$ exposure promotes ER signaling in premalignant mammary tissues from MMTV-ErbB2 mice. Since DMBA is reported to induce estrogen-dependent tumors (35), the present study examined the activation of ER $\alpha$ and ER target genes in mammary tissues from 15 -week-old mice. As presented in Fig. 4A, protein levels of both total ER $\alpha$ and phosphorylated 
Table III. DMBA induces chromosomal imbalance in mammary tumors from MMTV-ErbB2 mice.

A, Control tumors

\begin{tabular}{lcl}
\hline Sample & Karyotype & Chromosomal abnormality \\
\hline 08-MT-DO11T & $40, \mathrm{XX}$ & Normal \\
08-MT-DO12T & $40, \mathrm{XX}$ & Normal \\
08-MT-DO13T & $40, \mathrm{XX}$ & Normal \\
08-MT-DO14T & $40, \mathrm{XX}$ & Normal \\
08-MT-DO15T & $40, \mathrm{XX}$, add(4)(E2)[3]/41, XX, $+5[2] / 40, \mathrm{XX}[15]$ & Addition, trisomy \\
08-MT-DO18T & $40, \mathrm{XX}$ & Normal \\
\hline
\end{tabular}

B, DMBA tumors

\begin{tabular}{|c|c|c|c|}
\hline Sample & Karyotype & Chromosomal abnormality & Pattern \\
\hline 08-MT-CT-1 & $41, \mathrm{XX},+2[9] / 40, \mathrm{XX}[9]$ & Trisomy & Mosaic \\
\hline 08-МТ-СТ-2 & $40, \mathrm{XX}$ & Normal & \\
\hline 08-MT-CT-3 & $40, \mathrm{XX}$ & Normal & \\
\hline 08-MT-CT-4 & $41, X X,+3[14] / 40, X X[4]$ & Trisomy & Mosaic \\
\hline 08-MT-СТ-5 & $39, \mathrm{XX}, \operatorname{del}(4)(\mathrm{C} 2 \mathrm{D} 1)[2] / 40, \mathrm{XX}[15]$ & Deletion & Mosaic \\
\hline 08-MT-СТ-6 & $40, \mathrm{XX}$ & Normal & \\
\hline
\end{tabular}

Cell cycle arrest was induced in primary tumor cells from control and DMBA-treated MMTV-ErbB2 mice ( $\mathrm{n}=6$ mice per treatment) with Colcemid for $1 \mathrm{~h}$. Then, karyotyping was performed to examine chromosomal alterations. DMBA, DMBA, 7,12-dimethylbenz[a]anthracene.

$\mathrm{ER} \alpha$ were significantly increased in the premalignant mammary glands from DMBA-treated mice, whereas protein levels of ER $\beta$ remained unaltered. Consistently, the protein expression levels of c-Myc, Cyclin D1, and B cell lymphoma-2 (Bcl-2), which are downstream targets of the ER pathway, were also increased in DMBA-exposed mammary gland tissues. To further verify the activation of the ER pathway, mRNA levels of several ER target genes were detected. Results indicated that the transcription of ESRl (ER $\alpha), M Y C$ (c-Myc), CCNDI (Cyclin D1), and JUN (c-Jun) were significantly increased in the mammary tissues from the DMBA-treated mice, compared with control mice (Fig. 4B). These data substantiate that DMBA stimulates ER-mediated gene transcription in the premalignant mammary tissues.

DMBA induces chromosomal imbalance in mammary tumors from MMTV-ErbB2 mice. Previous reports have revealed that ErbB2-mediated carcinogenesis in MMTV-ErbB2 mice involves chromosome instability $(36,37)$. DMBA-induced DNA adducts and damage are critical factors in tumor initiation $(38,39)$. Therefore, the present study karyotyped chromosomes from the tumors to determine whether DMBA induces enhanced chromosomal alterations in MMTV-ErbB2 mice. As presented in Table III, one of the six analyzed tumors (16.7\%) from control mice displayed chromosomal abnormalities, which were associated with an addition in chromosome 4 and trisomy 5. Conversely, 50\% of the analyzed tumors from DMBA-treated mice exhibited abnormal chromosomes. These genetic lesions included trisomy 2, trisomy 3 , and a deletion in chromosome 4 . Overall, these results indicated that DMBA promotes chromosomal instability in the mammary tumors from MMTV-ErbB2 mice.

\section{Discussion}

Numerous studies have reported that breast carcinogenesis can be enhanced by environmental factors that have pro-estrogenic effects and increase gene susceptibility to chromosomal alterations, such as bisphenol $\mathrm{A}$ and DMBA (4,40-42). Understanding the molecular mechanisms of gene-environment interactions in breast carcinogenesis remains a significant challenge $(2,43)$. The present study investigated the effects of DMBA on ErbB2-mediated mammary tumor development in MMTV-ErbB2 transgenic mice. DMBA promotes mammary tumor development and the incidence of pulmonary metastasis $(44,45)$ in MMTV-ErbB2 mice. Prior to malignant transformation, DMBA also induces distinct mammary morphogenic changes as compared with control tissues $(14,46)$. The present study demonstrated that DMBA promoted ErbB2-mediated carcinogenesis through the enhanced activation of RTK and ER signaling. The synergistic effect of DMBA and ErbB2 deregulation on genomic instability also contributed to tumor development in this model. Since DMBA is a typical environmental carcinogen and ErbB2 is a clinically relevant oncogene associated with genetic predisposition to breast cancer, the present study provides important insight towards understanding gene-environment interactions associated with breast carcinogenesis.

In the present study, distinct changes in mammary morphogenesis were associated with DMBA exposure in 
MMTV-ErbB2 mice. In particular, DMBA exposure promoted ductal elongation beyond the lymph node, however resulted in mammary glands with less complex ductal structures than what is often induced by estrogenic factors (47). To potentially explain these morphological differences, previous reports have demonstrated that DMBA can upregulate genes involved in microtubule dynamics, while $\beta$-casein and transferrin, two key genes involved in mammary gland differentiation, are inhibited by DMBA (14). This evidence of DMBA-associated gene regulation may partially account for the increased ductal elongation and reduced ductal complexity observed in the study, which warrants further investigation.

Molecular analysis of premalignant mammary tissues from DMBA-exposed MMTV-ErbB2 mice provided mechanistic insight into DMBA-mediated carcinogenesis. It is known that the oncogenic driving force in MMTV-ErbB2 transgenic mice is increased activation of RTK signaling and its downstream pathways, such as PI3K/Akt and MAPK/Erk pathways. The results demonstrated that DMBA exposure enhanced the activation of EGFR, ErbB2, Akt, and Erk in the premalignant mammary tissues. These data indicated that DMBA has specific synergistic effects on ErbB2 activation and consequential tumorigenesis.

Consistent with previous reports indicating that DMBA induces estrogen-dependent mammary tumors in rats (35), it was revealed that DMBA significantly increased $E R \alpha$ protein phosphorylation/activation and ESR $1, M Y C, C C N D 1$, and $J U N$ mRNA levels in the premalignant mammary glands. Previous reports also corroborate that $C C N D 1$ upregulation is associated with carcinogen-induced mammary tumor development (14). Increased expression levels of Cyclin D1 and c-Myc are crucial in the carcinogenic role of DMBA (11). The present study additionally demonstrated that $C C N D 1 \mathrm{mRNA}$ levels were significantly upregulated in mammary tumors from the DMBA-exposed mice (data not shown), which is consistent with previous reports regarding Cyclin D1 regulation as a major contributor to DMBA-mediated tumor development in other model systems. Furthermore, concurrent activation of EGFR/ErbB2 and ER pathways accompanied by increased ER-targeted gene transcription suggests the activation of crosstalk between the RTK and ER signaling pathways. In the context of DMBA-induced oncogenic signaling activation, the findings underscore the role of EGFR/ErbB2-ER signaling crosstalk in DMBA-promoted mammary tumor development in MMTV-ErbB2 mice, which also sheds light on potential mechanisms of DMBA-mediated tumor development in other model systems.

DNA damage and genomic instability are essential components of DMBA-associated carcinogenic activities $(38,39)$. Previous reports have also demonstrated that genomic instability is involved in mammary tumor development in MMTV-ErbB2 mice (30). In the present study, mammary tumors from both control and DMBA-exposed MMTV-ErbB2 mice displayed chromosomal imbalance. Notably, it was revealed that the occurrence of genetic lesions/abnormalities in ErbB2-overexpressing mammary tumors from DMBA-treated mice was significantly increased compared with tumors from control mice, suggesting that enhanced genomic instability contributes to the accelerated tumor development in this model.
Results from the present study support the use of the MMTV-ErbB2 transgenic mouse model to investigate the interactions of environmental factors and genetic predisposition that contribute to breast carcinogenesis. Previous reports exploring the effects of DMBA on mammary tumor development in other transgenic models, such as MMTV-TGF $\alpha$ transgenic mice, provide fundamental support regarding the synergistic effects of genetic and environmental factors on carcinogenesis (48). Since ErbB2 overexpression is detected in approximately one-third of invasive breast cancers and more than half of ductal carcinoma in situ $(15,16)$, studies focusing on the effects of environmental carcinogens, including DMBA and other PAHs, on ErbB2-mediated carcinogenesis are of significant clinical relevance.

In conclusion, the establishment of this model system will facilitate future studies investigating the association between ErbB2-mediated breast cancer risk and environmental exposures, which will ultimately lead to effective strategies for the prevention of ErbB2-overexpressing breast cancers.

\section{Acknowledgements}

Not applicable.

\section{Funding}

This work was supported in part by the American Cancer Society (grant no. RSG-08-138-01-CNE), the National Institute of Environmental Health Sciences (grant no. R21ES025337), the National Cancer Institute (grant no. 5U54CA156735), and the National Institute on Alcohol Abuse and Alcoholism (grant no. U54 AA019765) to XY.

\section{Availability of data and materials}

All data generated or analyzed during this study are included in this published article or are available from the corresponding author on reasonable request.

\section{Authors' contributions}

ZM, YMK, SY, YJ, ABP, and XC performed the experiments and animal care; SDK performed histopathology; EWH, XC, and $\mathrm{XY}$ wrote the manuscript; EWH, SL, and XY performed data analysis and interpretation; XF, SL, and XY conceived and designed the experiments.

\section{Ethics approval and consent to participate}

All animal procedures were approved by the University of Oklahoma Health Sciences Center Institutional Animal Care and Use Committee (Oklahoma City, OK, USA).

\section{Patient consent for publication}

Not applicable.

\section{Competing interests}

The authors declare that they have no competing interests. 


\section{References}

1. Nowell SA, Ahn J and Ambrosone CB: Gene-nutrient interactions in cancer etiology. Nutr Rev 62: 427-438, 2004.

2. Song M, Lee KM and Kang D: Breast cancer prevention based on gene-environment interaction. Mol Carcinog 50: 280-290, 2011.

3. Chia KS: Gene-environment interactions in breast cancer. Novartis Found Symp 293: 143-150; discussion 150-155, 181-183, 2008.

4. Rubin BS: Bisphenol A: An endocrine disruptor with widespread exposure and multiple effects. J Steroid Biochem Mol Biol 127: 27-34, 2011.

5. Belpomme D, Irigaray P, Hardell L, Clapp R, Montagnier L, Epstein S and Sasco AJ: The multitude and diversity of environmental carcinogens. Environ Res 105: 414-429, 2007.

6. Schell LM, Burnitz KK and Lathrop PW: Pollution and human biology. Ann Hum Biol 37: 347-366, 2010.

7. Majkova Z, Toborek M and Hennig B: The role of caveolae in endothelial cell dysfunction with a focus on nutrition and environmental toxicants. J Cell Mol Med 14: 2359-2370, 2010

8. Rybicki BA, Nock NL, Savera AT, Tang D and Rundle A: Polycyclic aromatic hydrocarbon-DNA adduct formation in prostate carcinogenesis. Cancer Lett 239: 157-167, 2006.

9. Singhal R, Shankar K, Badger TM and Ronis MJ: Estrogenic status modulates aryl hydrocarbon receptor-mediated hepatic gene expression and carcinogenicity. Carcinogenesis 29: 227-236, 2008.

10. Mehta RG, Naithani R, Huma L, Hawthorne M, Moriarty RM McCormick DL, Steele VE and Kopelovich L: Efficacy of chemopreventive agents in mouse mammary gland organ culture (MMOC) model: A comprehensive review. Curr Med Chem 15: 2785-2825, 2008.

11. Currier N, Solomon SE, Demicco EG, Chang DL, Farago M, Ying $\mathrm{H}$, Dominguez I, Sonenshein GE, Cardiff RD, Xiao ZX, et al: Oncogenic signaling pathways activated in DMBA-induced mouse mammary tumors. Toxicol Pathol 33 726-737, 2005.

12. Nebert DW, Petersen DD and Fornace AJ, Jr: Cellular responses to oxidative stress: The [Ah] gene battery as a paradigm. Environ Health Perspect 88: 13-25, 1990.

13. Rundle A, Tang D, Hibshoosh H, Estabrook A, Schnabel F, Cao W, Grumet S and Perera FP: The relationship between genetic damage from polycyclic aromatic hydrocarbons in breast tissue and breast cancer. Carcinogenesis 21: 1281-1289, 2000

14. Papaconstantinou AD, Shanmugam I, Shan L, Schroeder IS Qiu C, Yu M and Snyderwine EG: Gene expression profiling in the mammary gland of rats treated with 7,12-dimethylbenz[a] anthracene. Int J Cancer 118: 17-24, 2006

15. Liu E, Thor A, He M, Barcos M, Ljung BM and Benz C: The HER2 (c-erbB-2) oncogene is frequently amplified in in situ carcinomas of the breast. Oncogene 7: 1027-1032, 1992.

16. Jardines L, Weiss M,FowbleB and Greene M:neu(c-erbB-2/HER2) and the epidermal growth factor receptor (EGFR) in breast cancer. Pathobiology 61: 268-282, 1993.

17. Hynes NE and Stern DF: The biology of erbB-2/neu/HER-2 and its role in cancer. Biochim Biophys Acta 1198: 165-184, 1994.

18. Citri A and Yarden Y: EGF-ERBB signalling: Towards the systems level. Nat Rev Mol Cell Biol 7: 505-516, 2006.

19. Guy CT, Webster MA, Schaller M, Parsons TJ, Cardiff RD and Muller WJ: Expression of the neu protooncogene in the mammary epithelium of transgenic mice induces metastatic disease. Proc Natl Acad Sci USA 89: 10578-10582, 1992.

20. Bouchard L, Lamarre L, Tremblay PJ and Jolicoeur P: Stochastic appearance of mammary tumors in transgenic mice carrying the MMTV/c-neu oncogene. Cell 57: 931-936, 1989.

21. Marcotte R and Muller WJ: Signal transduction in transgenic mouse models of human breast cancer-implications for human breast cancer. J Mammary Gland Biol Neoplasia 13: 323-335, 2008.

22. Hutchinson JN and Muller WJ: Transgenic mouse models of human breast cancer. Oncogene 19: 6130-6137, 2000.

23. Yang X, Yang S, McKimmey C, Liu B, Edgerton SM, Bales W, Archer LT and Thor AD: Genistein induces enhanced growth promotion in ER-positive/erbB-2-overexpressing breast cancers by ER-erbB-2 cross talk and p27/kip1 downregulation. Carcinogenesis 31: 695-702, 2010.

24. Yang X, Edgerton SM, Kosanke SD, Mason TL, Alvarez KM Liu N, Chatterton RT, Liu B, Wang Q, Kim A, et al: Hormonal and dietary modulation of mammary carcinogenesis in mouse mammary tumor virus-c-erbB-2 transgenic mice. Cancer Res 63 : 2425-2433, 2003.

25. Warin R, Chambers WH, Potter DM and Singh SV: Prevention of mammary carcinogenesis in MMTV-neu mice by cruciferous vegetable constituent benzyl isothiocyanate. Cancer Res 69: 9473-9480, 2009.
26. Shen K and Novak RF: DDT stimulates c-erbB2, c-met, and STATS tyrosine phosphorylation, Grb2-Sos association, MAPK phosphorylation, and proliferation of human breast epithelial cells. Biochem Biophys Res Commun 231: 17-21, 1997.

27. Shan L, Yu M and Snyderwine EG: Global gene expression profiling of chemically induced rat mammary gland carcinomas and adenomas. Toxicol Pathol 33: 768-775, 2005.

28. Shan L, He M, Yu M, Qiu C, Lee NH, Liu ET and Snyderwine EG: cDNA microarray profiling of rat mammary gland carcinomas induced by 2-amino-1-methyl-6-phenylimidazo[4,5-b]pyridine and 7,12-dimethylbenz[a]anthracene. Carcinogenesis 23 : $1561-1568,2002$

29. Livak KJ and Schmittgen TD: Analysis of relative gene expression data using real-time quantitative PCR and the $2^{-\Delta \Delta C \mathrm{~T}}$ method. Methods 25:402-408, 2001.

30. Kim YM, Ma Z, Lee S, Lee J, Li S and Yang X: Trisomy chromosome 5 is a recurrent cytogenetic lesion in mammary tumors from parous MMTV-erbB-2 transgenic mice. Oncol Lett 2: 1077-1081, 2011.

31. de Oliveira Andrade F, Fontelles CC, Rosim MP, de Oliveira TF, de Melo Loureiro AP, Mancini-Filho J, Rogero MM, Moreno FS, de Assis S, Barbisan LF, et al: Exposure to lard-based high-fat diet during fetal and lactation periods modifies breast cancer susceptibility in adulthood in rats. J Nutr Biochem 25: 613-622, 2014.

32. Qin LQ, Xu JY, Tezuka H, Wang PY and Hoshi K: Commercial soy milk enhances the development of 7,12-dimethylbenz(a) anthracene-induced mammary tumors in rats. In Vivo 21: 667-671, 2007.

33. Qi C, Lan H, Ye J, Li W, Wei P, Yang Y, Guo S, Lan T, Li J, Zhang Q, et al: Slit2 promotes tumor growth and invasion in chemically induced skin carcinogenesis. Lab Invest 94: 766-776, 2014.

34. Muller WJ, Sinn E, Pattengale PK, Wallace R and Leder P: Single-step induction of mammary adenocarcinoma in transgenic mice bearing the activated c-neu oncogene. Cell 54: 105-115, 1988.

35. Mohibi S, Mirza S, Band H and Band V: Mouse models of estrogen receptor-positive breast cancer. J Carcinog 10: 35, 2011.

36. Cool M, Depault F and Jolicoeur P: Fine allelotyping of Erbb2-induced mammary tumors in mice reveals multiple discontinuous candidate regions of tumor-suppressor loci. Genes Chromosomes Cancer 45: 191-202, 2006.

37. Liu S, Liu W, Jakubczak JL, Erexson GL, Tindall KR, Chan R, Muller WJ, Adhya S, Garges S and Merlino G: Genetic instability favoring transversions associated with ErbB2-induced mammary tumorigenesis. Proc Natl Acad Sci USA 99: 3770-3775, 2002

38. Daniel FB and Joyce NJ: DNA adduct formation by 7 , 12-dimethylbenz[a]anthracene and its noncarcinogenic 2-fluoro analogue in female Sprague-Dawley rats. J Natl Cancer Inst 70: $111-118,1983$

39. Russo IH and Russo J: Mammary gland neoplasia in long-term rodent studies. Environ Health Perspect 104: 938-967, 1996.

40. Umekita Y, Souda M, Hatanaka K, Hamada T, Yoshioka T, Kawaguchi $\mathrm{H}$ and Tanimoto A: Gene expression profile of terminal end buds in rat mammary glands exposed to diethylstilbestrol in neonatal period. Toxicol Lett 205: 15-25, 2011.

41. Hsu PY, Deatherage DE, Rodriguez BA, Liyanarachchi S, Weng YI, Zuo T, Liu J, Cheng AS and Huang TH: Xenoestrogen-induced epigenetic repression of microRNA-9-3 in breast epithelial cells. Cancer Res 69: 5936-5945, 2009.

42. Lamartiniere CA, Jenkins S, Betancourt AM, Wang J and Russo J: Exposure to the endocrine disruptor bisphenol a alters susceptibility for mammary cancer. Horm Mol Biol Clin Investig 5: 45-52, 2011.

43. Wogan GN, Hecht SS, Felton JS, Conney AH and Loeb LA: Environmental and chemical carcinogenesis. Semin Cancer Biol 14: 473-486, 2004.

44. Medina D: Chemical carcinogenesis of rat and mouse mammary glands. Breast Dis 28: 63-68, 2007.

45. Plante I, Stewart MK, Barr K, Allan AL and Laird DW: Cx43 suppresses mammary tumor metastasis to the lung in a $\mathrm{Cx} 43$ mutant mouse model of human disease. Oncogene 30: 1681-1692, 2011.

46. Russo $\mathrm{J}$ and Russo IH: Influence of differentiation and cell kinetics on the susceptibility of the rat mammary gland to carcinogenesis. Cancer Res 40: 2677-2687, 1980.

47. Markey CM, Luque EH, Munoz De Toro M, Sonnenschein C and Soto AM: In utero exposure to bisphenol A alters the development and tissue organization of the mouse mammary gland. Biol Reprod 65: 1215-1223, 2001.

48. Coffey RJ Jr, Meise KS, Matsui Y, Hogan BL, Dempsey PJ and Halter SA: Acceleration of mammary neoplasia in transforming growth factor alpha transgenic mice by 7,12-dimethylbenzanthracene. Cancer Res 54: 1678-1683, 1994. 